\title{
Prediction of transonic buffet around a wing with flap
}

\author{
G. Barbut ${ }^{1}$, M. Braza ${ }^{1}$, Y. Hoarau ${ }^{2}$, G. Barakos ${ }^{3}$, A. Sévrain ${ }^{1}$, J.B. Vos $^{4}$ \\ ${ }^{1}$ Institut de Mécanique des Fluides de Toulouse, France, corresponding \\ author : barbut@imft.fr \\ ${ }^{2}$ Institut de Mécanique des Fluides et de Solides de Strasbourg, France \\ ${ }^{3}$ University of Liverpool, UK \\ ${ }^{4}$ CFS Engineering, Switzerland
}

\begin{abstract}
The present study presents numerical simulations and turbulence modelling of the flow around a NACA0012 airfoil including a deflected aileron. The results are compared with experiments that have been performed in the N-3 wind tunnel of the Institute of Aviation (IoA), Warsaw. The experiment focused on unsteady flow characteristics and buffet phenomena arising as the result of the transonic shock wave / boundary layer interaction (SWBLI). The transonic buffet is a natural and self-sustaining oscillation of the shock wave and separated flow region, caused by pressure fluctuation. The first objective is to capture the transonic buffet unsteadiness by means of URANS and DES turbulence modelling approaches. Secondly, the periodic flap oscillation has been used to modify the oscillation amplitudes towards an outlook of attenuation of the transonic buffet.
\end{abstract}

\section{Introduction}

Within the UFAST (Unsteady Effects in Shock Wave induced separation) program, it has been shown through its main test-cases, that URANS approaches were not successful to capture unsteady shock wave boundary layer interaction, SWBLI for internal geometries, whereas they were more successful for external flow configurations. These facts are reported in the UFAST books, published also by Springer in 2010. The DES approaches were more successful than URANS for internal flow configurations, whereas they are comparable to URANS for external flow configurations (example: the INCAS-biconvex test-case), where also transonic buffet occurs. In the present study, the transonic buffet over the NACA0012 wing with deflected aileron is investigated. First, the natural unsteadiness is examined as a self-sustaining oscillation of the shock wave and of the separated flow region, caused by pressure fluctuation (Seegmiller et al, 1978). A first objective of the present study is to capture the transonic buffet unsteadiness by means of URANS and DES turbulence modelling approaches. A second objective is to perform a sensitivity study concerning the effect of the periodic flap oscillation, that is used to modify the oscillation amplitudes. This goal is related to 
current and prospective studies for attenuation of the transonic buffet by deflected ailerons. In the present study, the URANS-Spalart-Allmaras (SA), the URANS- $k$ $\omega$, the DES-SA and the DDES - SA turbulence modelling approaches have been used.

\section{Test-case Description}

\section{Experimental set-up}

The NACA0012 airfoil model equipped with $22.6 \%$ of airfoil chord flap and modified gap as shown in Fig. 1 was tested. The model's chord is equal to $180 \mathrm{~mm}$ and flap length is $40.69 \mathrm{~mm}$. The axis of rotation of the main part of the model is at $35 \%$ of the chord. There is a smaller, $0.3 \mathrm{~mm}$ gap between airfoil main part and flap leading edge. The flap leading edge radius is $4.69 \mathrm{~mm}$. The axis of the aileron/ flap rotation is at $80 \%$ of the airfoil model chord. The span of the model is $596 \mathrm{~mm}$.

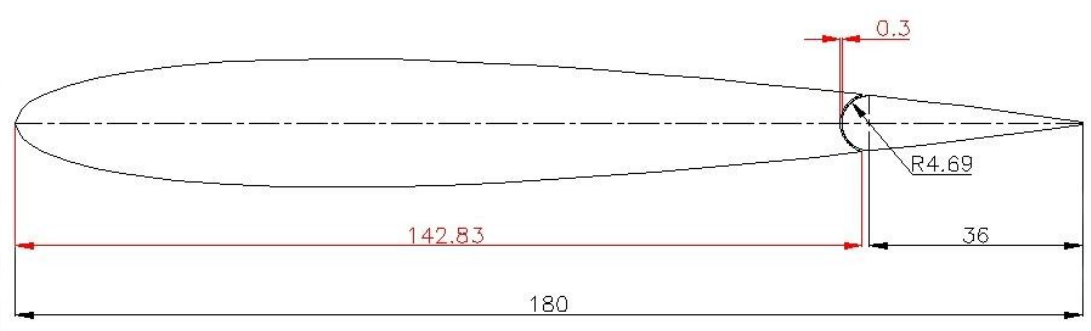

Fig. 1. The geometry of the NACA 0012 model equipped with the flap.

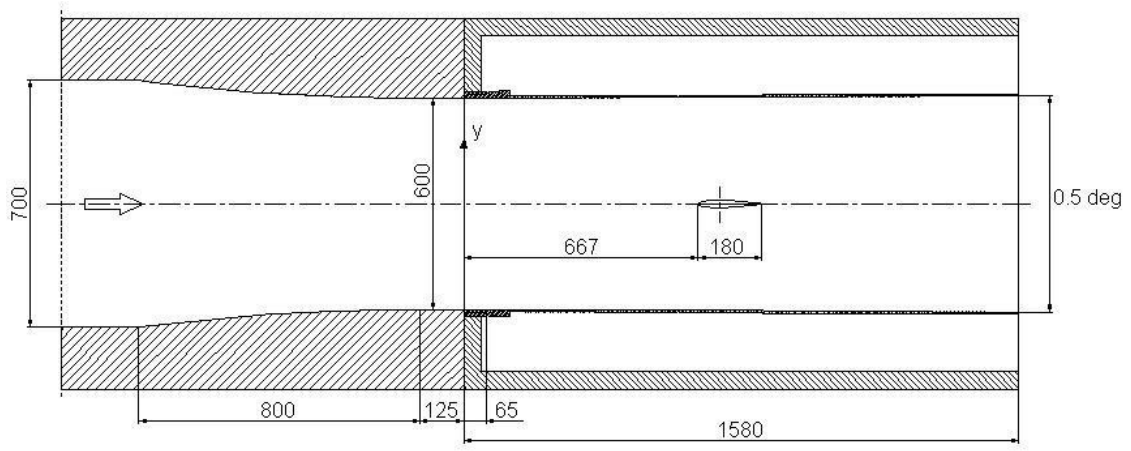

Fig. 2. Geometry of the test section, IoA N-3 wind tunnel with the NACA 0012 flapped model. 
The computations have been carried by using the real wind-tunnel 3D geometry (fig.2). The angle of attack is $5^{\circ}$. The Mach number is $\mathrm{M}=0.73$ and Reynolds number $\operatorname{Re}=2.69 \cdot 10^{6}$. The amplitude of the flap deflection angle up to $\Delta \delta_{\phi}= \pm 2^{\circ}$. The oscillation frequency of the flap, $f_{f}=100 \mathrm{~Hz}$ and $30 \mathrm{~Hz}$ (close to the buffet frequency and one third of the buffet frequency, respectively).

The experimental signals display a strong chaotic character, interspersed by peaks of more organised events in respect to the buffet phenomenon (figure 3). This complex behaviour was enhanced by the fact that the upper and lower walls are not porous but solid, fixed walls. IMFT has applied advanced signal processing techniques, as autoregressive modelling (AR), Burg algorithm as described in Marple (1987) and Morlet's wavelets analysis (Grossmann \& Morlet-1984), to extract the organised part and to assess the contribution of the organised and random parts in the physical process.

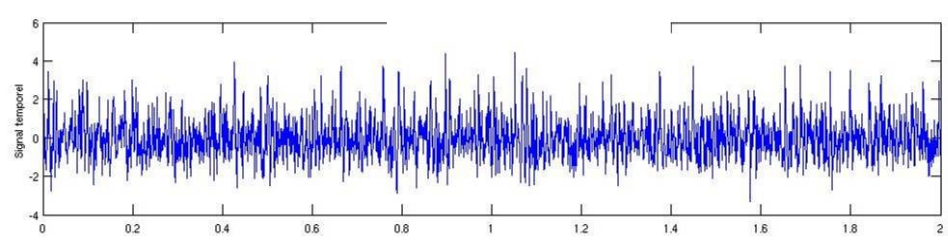

Spectrum CT Burg, order $=30$, duration $=0.05 \mathrm{~s}$, step $=0.0031$

Freq.

$(\mathrm{Hz})$

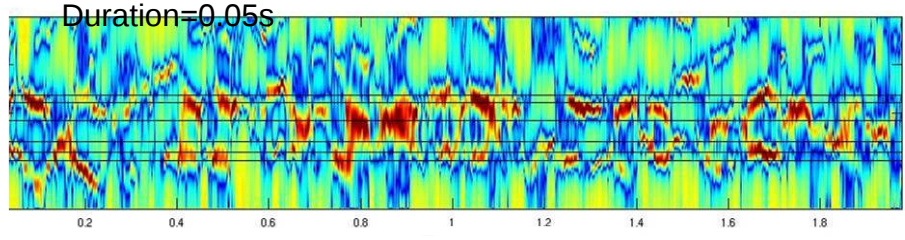

Freq.

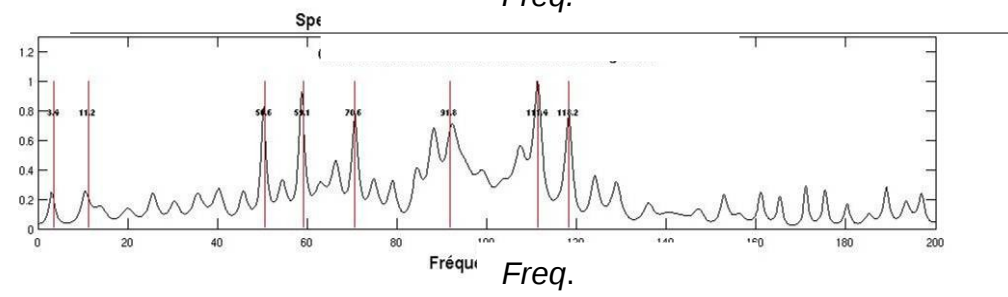

Fig. 3. Experimental signal, surface pressure at $50 \%$ of chord versus time, (up); autoregressive modeling (AR)-(middle), FFT spectrum (down), $\mathrm{M}=0.7, \alpha=6.8$, $\operatorname{Re}=2.63 \times 10^{6}$ 
The AR model indicates organisation of the flow according to a bi-modal pattern with a predominant frequency of order $95-100 \mathrm{~Hz}$, corresponding to the buffet phenomenon and a first sub-harmonic of order $50 \mathrm{~Hz}$. The lower sub-figure in fig. 3 shows the FFT analysis that provides a multitude of frequencies in the present case. A similar behaviour is obtained from the Morlet's wavelet analysis, according to the previously mentioned bi-modal pattern. In case of forced oscillation of the flap by a frequency $\mathrm{f}=35 \mathrm{~Hz}$ in the IoA experiment (Miller et al, 2009), the organisation of the signal shown by AR and by Morlet's wavelets indicates a shift of the organised motion around the forcing frequency $(35 \mathrm{~Hz})$, as well as the appearance of a first harmonic of this frequency, of order $70 \mathrm{~Hz}$. The signal processing of this forced case is not presented in the present paper.

Numerical simulation of the IoA test-case

The transonic flow case of the IoA was studied for clean and flapped wing configurations. From the very start, the influence of the wind tunnel walls was identified as a key problem and a special mesh was put together to allow for modelling of the walls and easy comparison with free-stream conditions. At the time this report was compiled very few experimental data were available for comparisons and the exact flow case conditions were not specified. For this purpose, the University of Liverpool (ULIV) and IMFT focussed on a set of cases and comparisons with test data were made. The numerical grids are provided by ULIV (fig. 4 up) with 2.65 million cells including 18 blocks and by IMFT that performed refinement on the upper and lower walls as well as an optimised grid around the gap (fig.4 down). The refinement in the upper and lower walls was done in respect of the $y+$ value (less than 1). IMFT final grids are of order 3.3 million and 3.75 million cells. A special attention has been attributed to correctly mesh the gap region and to provide CFD on a computational domain with the real wind-tunnel walls. The grid spacing in the mid-span is of order 0.03 . The DES computation has been active in the flow regions beyond the near-wall ones.

The resolution of the wing surface was higher than the side walls which were put at exactly the same location as in the experiment. Care was taken to allow adequate grid resolution near the front $20 \%$ of the NACA-0012 wing section. This would allow for shocks to be resolved and capture any separation due to shock/boundary-layer interaction. The work focussed at relatively high incidence angles (up to 6.8 degrees) for which buffet clearly appears and for this reason, the upper surface of the wing was also meshed at a finer level. In total 2.65 million cells were used and the baseline grid included 18 blocks at each span-wise station. The time step size used was $10^{-7}$ for the URANS and DES computations. Statistics were evaluated following the elimination of initial transient phases.. The statistics obtained for the DES would need to be checked for convergence by using longer sample sizes in a future study.

The numerical code used by ULIV is the PMB code (Barakos, 2009) and by IMFT the NSMB, (Navier-Stokes MultiBlock code) (Vos et al, 1998) in which IMFT, 
being part of the NSMB consortium, has implemented higher-order turbulence models.

In the present study, the employed turbulence models concerning URANS are the Spalart Almaras (SA) (1992) and the $k-\omega$ model (Wilcox 1994). Concerning Detached Eddy Simulation, the DES-Spalart Allmaras (DES-SA) (1997), the DDES-Spalart-Allmaras (DDES-SA) (2006) have been used.
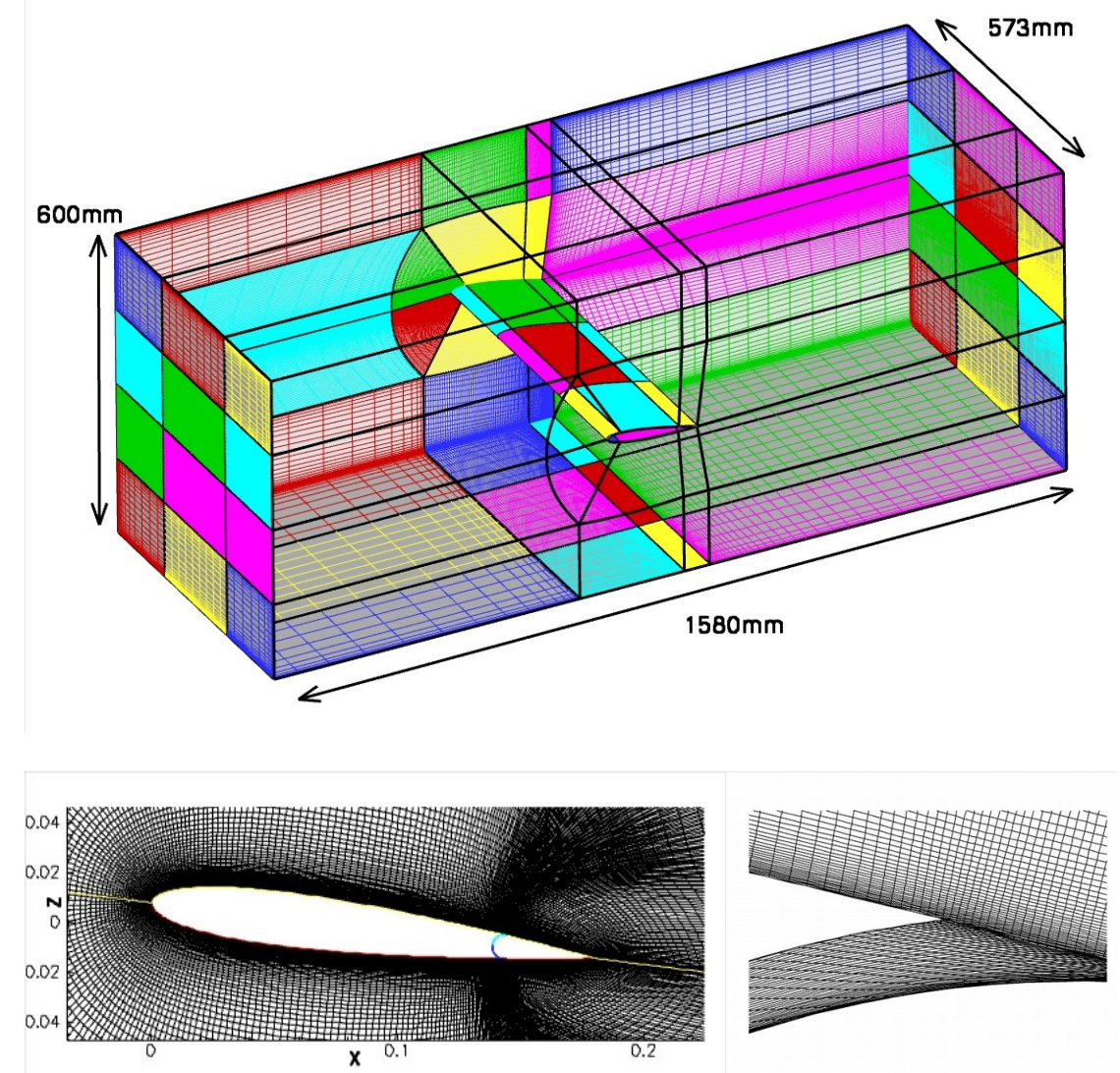

Fig. 4. View of the baseline mesh constructed by ULIV with ICEM-CFD Hexa. Up: overall domain configuration and block boundaries; down: adapted mesh by IMFT around the gap. 


\section{$5 \quad$ Investigation of Flow Conditions}

Figure 5 presents an overview of the flow configuration obtained for the wing with the tunnel walls present. The colour surface represents pressure that illustrates formation of a strong shock near the leading edge of the wing at a Mach number of 0.7 and 5 degrees of angle of attack. The strength of the shock is high enough to cause separation of the boundary layer and the flow near the side-walls of the tunnel is dominated by flow re-circulation regions. Near the mid-span of the wing, there is an extended region where the flow shows little spanwise variation. This suggests that a zero gradient condition could be imposed in this direction and a slice of the wing near the centre would be taken into account for the simulation. This approach would result in lower computational cost. However, the top and lower walls have a stronger effect and as was investigated, their effect must be accounted for. The flow reversal near the mid-plane of the wing and the walls is clearly visible in Figure 5, as well as the corner vortices.

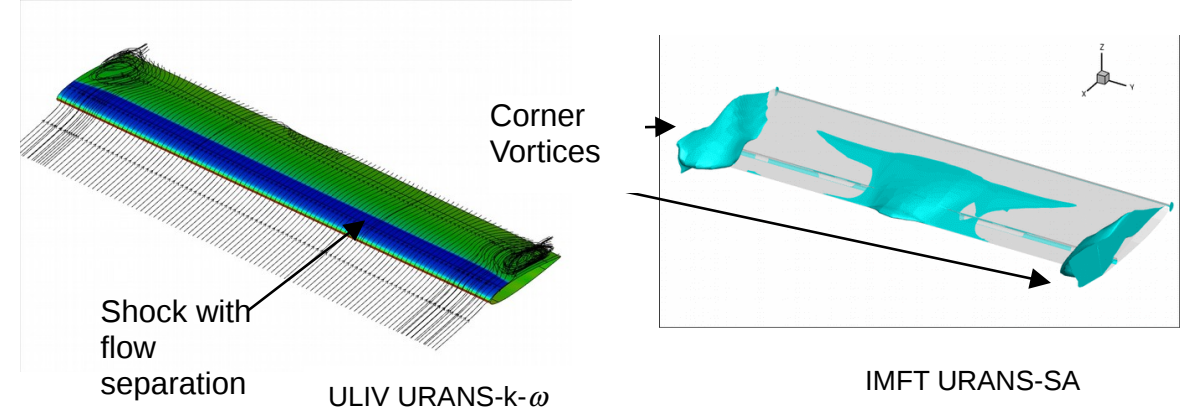

Fig. 5. Overview of flow configuration: flow recirculation and corner vortices near the side walls of the tunnel. Streamlines and iso contours of pressure coefficicient (left). Iso- $(U=0)$ velocity contours (right). IMFT: URANS-SA

The effect of the tunnel walls was studied. Computations with the upper and lower walls and with free-stream conditions result in different surface pressure coefficients Overall, the presence of the walls resulted in higher levels of surface pressure coefficient comparing with the non-confined case. Without the presence of the walls, the buffet onset is shifted to higher Mach numbers and angle-ofattack. This significant influence suggested that the upper and lower wind tunnel walls must be taken into account for the flow simulation.

A study of the spanwise conditions is presented on Figure 6. It can be seen that the full 3D configuration compares better with the experimental data than the computation where spanwise symmetry was imposed. It is therefore considered 
necessary to represent also the lateral walls in the simulation. An interesting observation is that the flow near the flap may be influenced by the exact geometry of the gap between the main element and the flap of the wing. The slotted flap configuration has a small effect near the trailing edge, fig. 6 (right).

To enhance the possibility of using a simplified geometry for actuation of the flap, slotted and blended flap configurations were used. The conditions were Mach number of 0.75 and Reynolds number of 2.81 million. The angle of attach of the wing was changed between 0 and five degrees (where the onset of the buffet was close). At the same time the flap was deflected by three degrees. For all cases, blended and slotted flaps were used. Flow visualisation is shown in Fig. 7 for the flow region near the slot. The conditions were selected so that the pressure difference between the lower and upper wing surfaces drives the flow through the gap. As a result a small flow distortion is shown. This flow distortion is also evident in the surface pressure coefficient plots shown in Fig 6 (right).
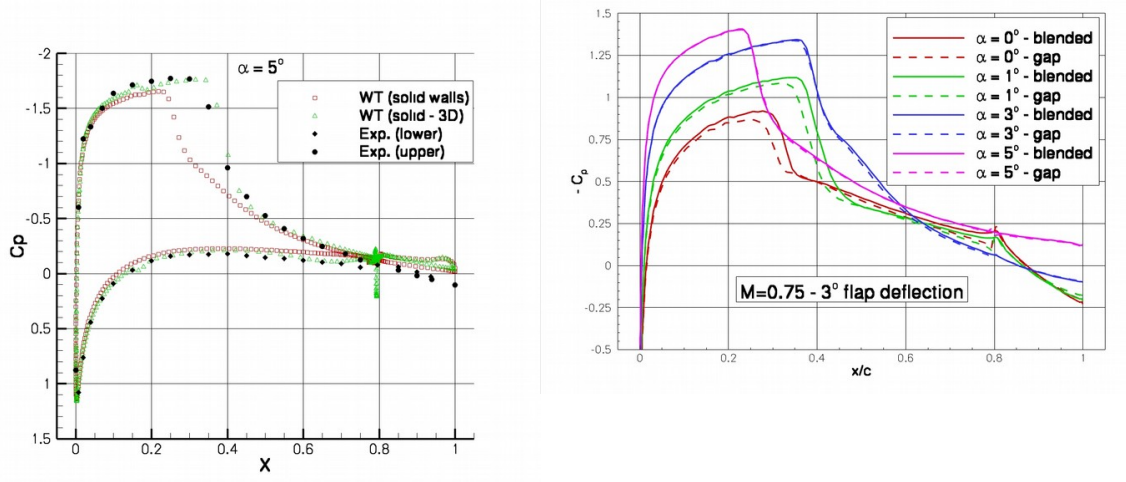

Fig. 6. Cp wall pressure coefficient compared with the experiment; axisymmetric assumption providing lower $\mathrm{Cp}$, versus full 3D WT geometry computation. Slotted mesh including the gap between main and flap elements (left). Comparison between blended and slotted flap configurations (right), $\mathrm{Re}=2.81 \times 10^{6}$

A zoom of the flow within the gap region is shown in figure 8, indicating the suction and pressure effects in the gap. Figure 9 shows IMFT computations concerning the time history of pressure on the wing surfaces, according to the refined mesh of fig. 4 (lower). The computations have been carried out during enough time to achieve several buffet cycles. The time-dependent pressure coefficients at two different chord positions (25\% and 50\%) are shown. In each figure, the pressure side and suction side positions are presented simultaneously. The suction side develops more irregular fluctuations due to the shock motion, especially at the $50 \%$ chord position. The pressure signals at each chord position are practically in phase opposition. Because of solving the phase-averaged Navier-Stokes equations in the URANS modelling, understood as a decomposition of the flow into coherent (resolved) and turbulent (modelled) motions, the high-frequency noise appearing in the experimental signals has been attenuated. 
Third Symposium on Hybrid RANS-LES Methods, 10-12 June 2009, Gdansk, Poland
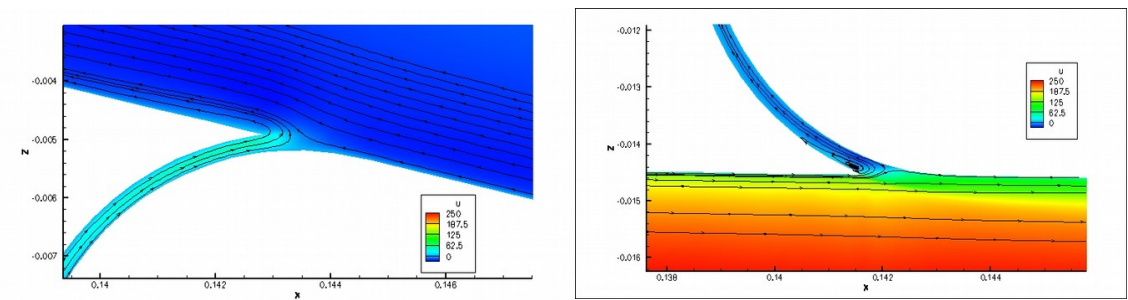

Fig. 8. Instantaneous iso- $U$ contours in the gap region
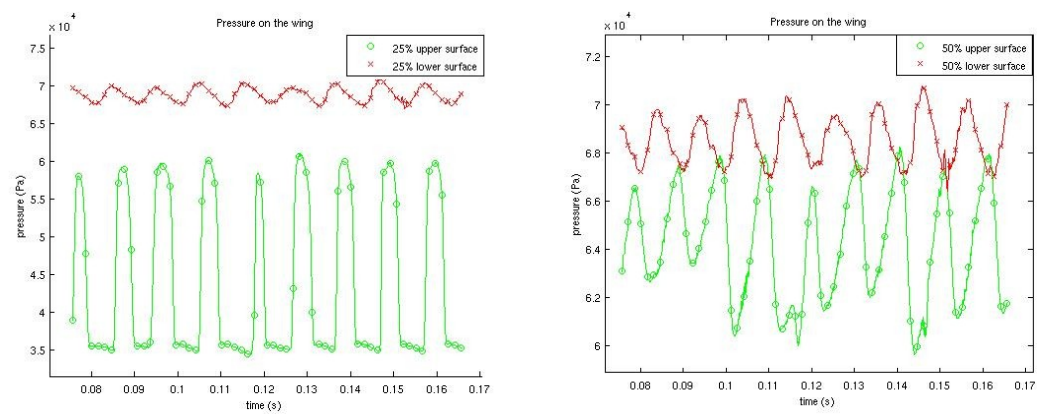
Fig. 9. Pressure signals, wing with gap, $\mathrm{M}=0.73$, $a=5^{\circ}$, Re $2.65 \times 10^{6}$, URANS-SA.

The buffet frequency obtained by the above IMFT results is of the order of $95 \mathrm{~Hz}$, in good agreement with ULIV results and with the physical experiment. It is recalled that the experimental data processing in respect of the buffet frequency has been performed later than the CFD evaluation of this frequency. Therefore, the present test-case was a 'blind' one in respect of the predominant frequency value. The results presented in Fig. 10 (left) show flow visualisation by means of instantaneous iso- $U$ contours at the median plane. The SWBLI is clearly illustrated and compared with the experimental Schlieren visualisation (fig. 11 left) of the IoA. Figure 10 (right) shows $r m s$ of the pressure field at the suction side. This illustrates the region affected by the shock motion. Figure 11 (right) shows an instantaneous field of the ratio $(\operatorname{grad} \rho / \rho), \rho$ being the density. This compares quite well with the instantaneous Schlieren visualisation.

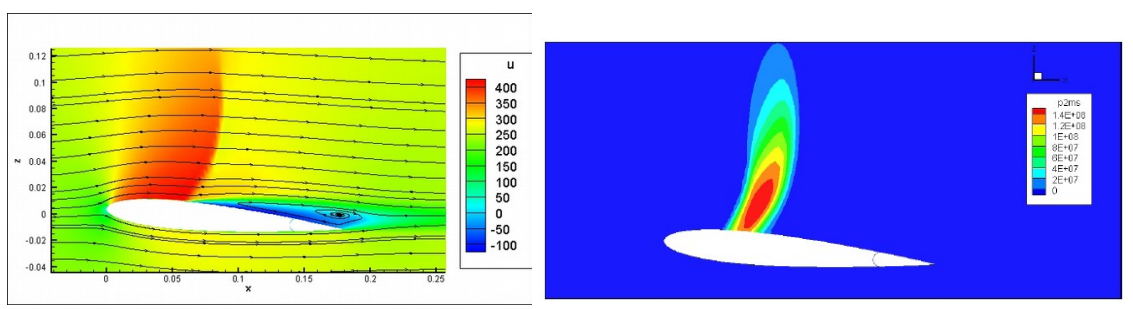

Fig.10. Left: Instantaneous Iso- $U$ contours at the medium span. Right: $r m s$ fluctuation of midspan pressure illustrating the compressibility effects in the shock region contours; URANS-SA.
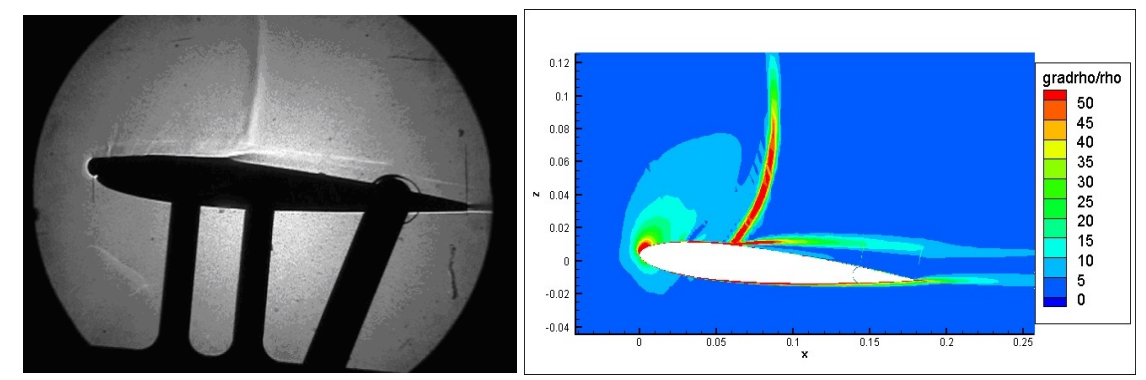

Fig.11. Left : IoA shadowgraph showing SWBLI on the NACA 0012 airfoil with undeflected flap at $\alpha=5^{\circ}$, Mach number $M=0.70$ and Reynolds number $\operatorname{Re}=2.69 \cdot 10^{6}$. Right: Instantaneous iso-contours of the ratio $(\operatorname{grad} \rho / \rho)$, $\rho$ being the density.

Furthermore, comparisons of the CFD pressure coefficient with the experiment are presented on fig. 13. ULIV have used the DES-SA modelling, where IMFT has 
used the DDES-SA (Delayed DES). A quite good comparison of the mean wall pressure coefficient is achieved, especially by ULIV. The difference between the two DES could however be a consequence of the short preliminary statistical sample computed.
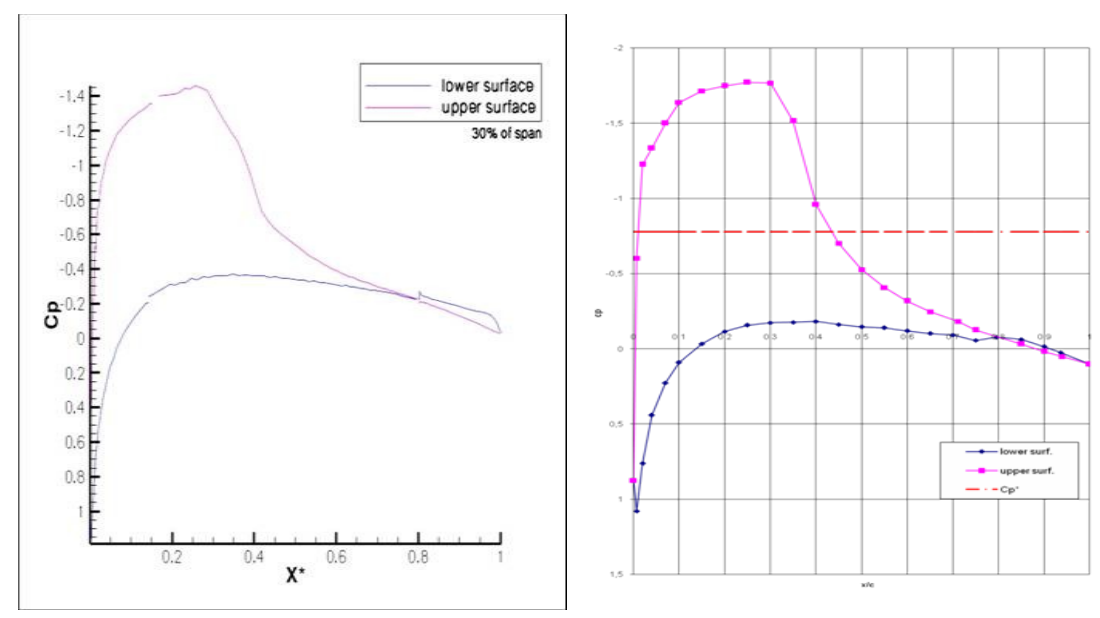

Fig. 12. Left: Time-averaged wall pressure coefficient at 30\% spanwise position (CFD). Right: experimental wall pressure coefficient (right), URANS - SA model.
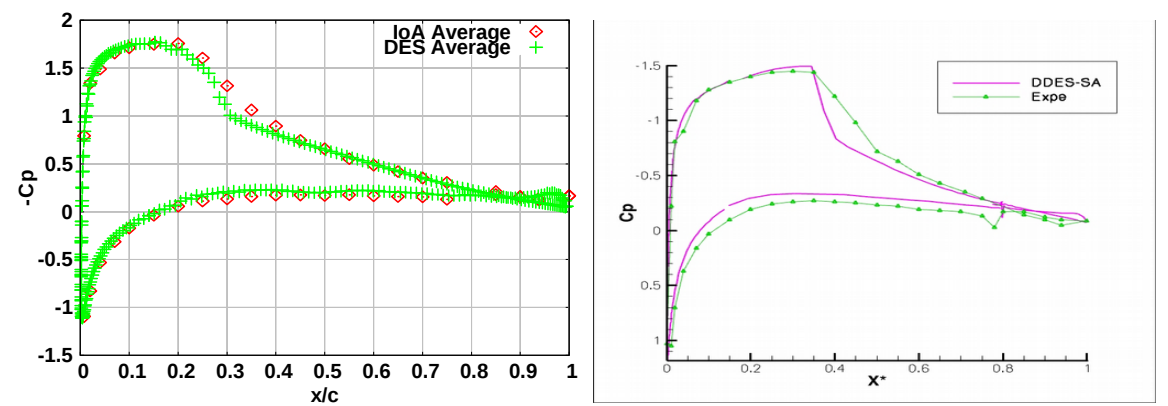

Fig. 13. Comparison of the mean wall pressure coefficient with the experiment: Left: ULIV; right IMFT.

Figure 14 shows URANS-SA and DDES computations at 50\% of chord, for the suction and pressure sides. The URANS field has been used as initial condition for the DDES computations. The two signals are practically in phase opposition by 
$180^{\circ}$. The buffet frequency is clearly pointed out by both approaches. Longer computational times should be used for the DDES approach to capture a more rich statistical content.
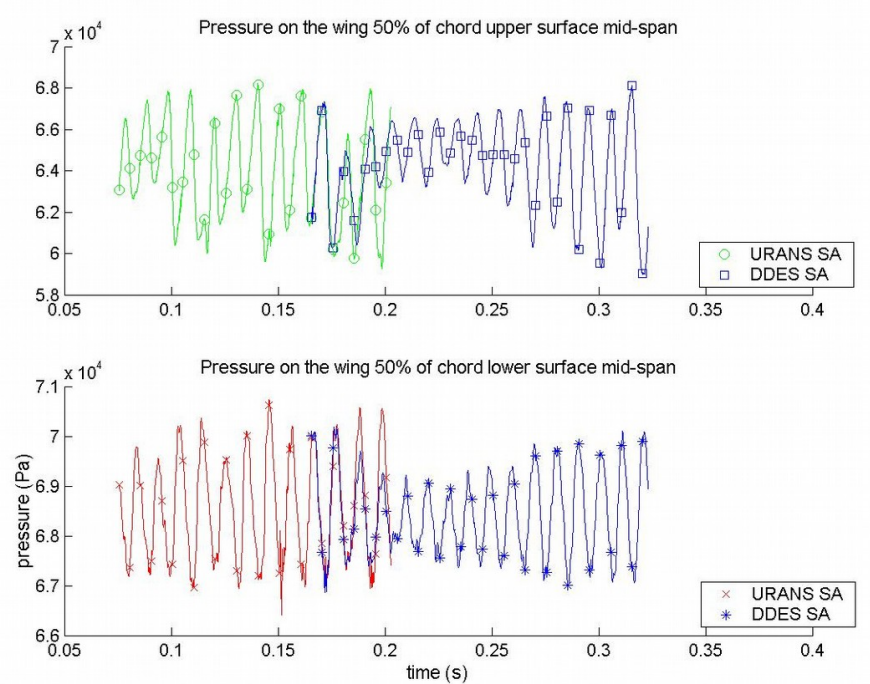

Fig. 14. Surface pressure signal at $50 \%$ of chord, URANS-SA and DDES-SA; up: suction side; down: pressure side.

\section{$6 \quad$ Flap actuation for flow control}

The effect of the flap actuation has been studied in two cases in the UFAST program, with actuation frequency of $30 \mathrm{~Hz}$, close to one third of the buffet frequency and a frequency of $100 \mathrm{~Hz}$, closed to the buffet frequency respectively. The flap was oscillating at an amplitude of 2 degrees.

After an initial time where the flow adapts from a partially-converged steady-state to buffet, the loads show an almost periodic response.
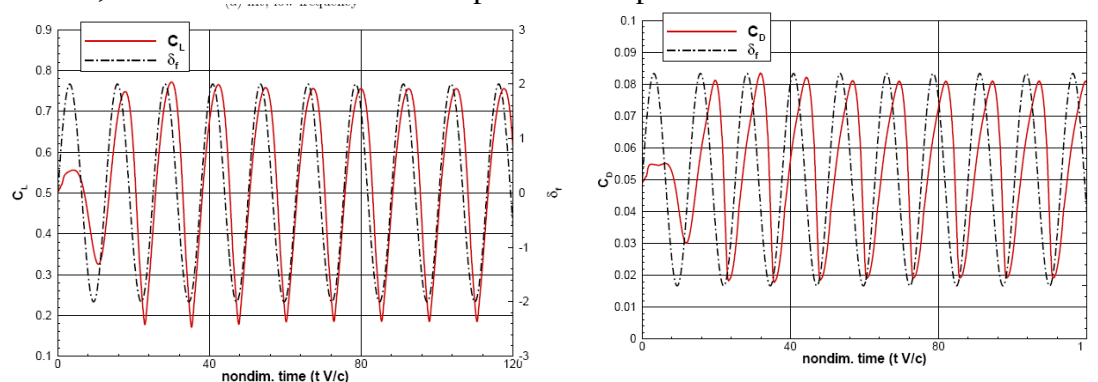
Fig. 15: Flap oscillation of 2 degrees at the buffet frequency; left: lift coefficient and flap angle, $\delta_{f}$, versus time. Right: drag coefficient and $\delta_{f} . \mathrm{M}=0.7, \mathrm{Re}=2.63 \times 10^{6}$ (ULIV).

Figure 15 shows the lift and drag time-dependent coefficients with and without flap deflection. It is noticeable that the present actuation does not considerably attenuate the buffet frequency.

The results of Figure 16 present comparison of the controlled and uncontrolled cases with blended and slotted flaps. In the case of actuation with a frequency very close to the buffet natural frequency, it is found that the amplitudes of the oscillation increase. In the case of actuation with a third of the buffet frequency, these amplitudes decrease. Furthermore, the flap motion appears introducing extra frequencies in the flow and induce loads modulation.
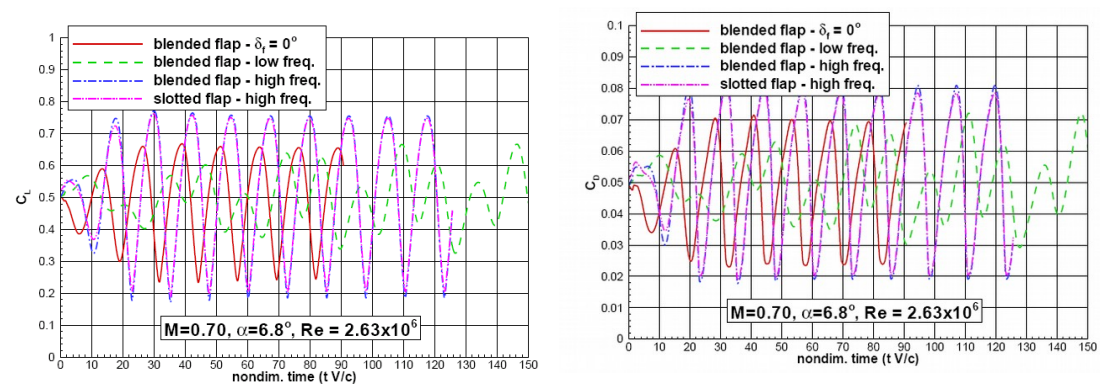

Fig. 16. Comparison of controlled and uncontrolled cases (flow parameters as in previous figure), ULIV, with two oscillation frequencies.

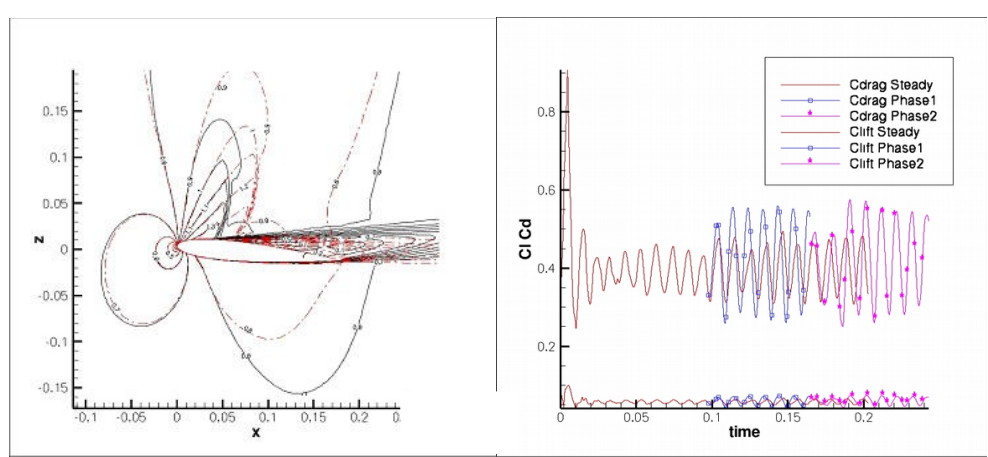

Fig. 17. Left: mid span iso-Mach lines at two different phases of the natural buffet oscillations (maximum and minimum of the Clift), corresponding to the starting of flap actuation. Right: Flap actuation at forcing frequency of $100 \mathrm{~Hz}$ and a deflection of $\pm 2^{\circ}$. In legend, "steady" means fixed flap. IMFT - URANS-SA.

Figure 17 shows the influence of forcing at $100 \mathrm{~Hz}$ (very close to the natural buffet frequency), actuated at two different phases (maximum and minimum of the Clift 
coefficient). The computations have been carried out by IMFT by URANS-SA. Both cases lead to an increase of lift coefficient amplitudes, as was pointed out by the computations of ULIV. This can be explained as a 'resonance' lock-in between the buffet frequency and the forcing frequency $(100 \mathrm{~Hz})$, created by the flap motion. Figure 18 shows the 3D modulation of the shock area along the span in the first case (flap oscillations starting at an instant of maximum Clift).

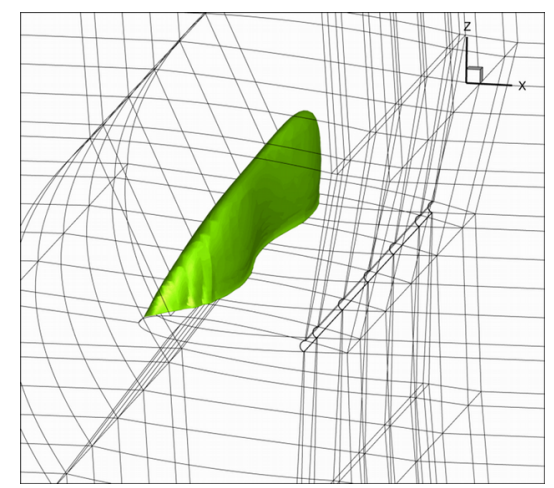

Fig. 18. Instantaneous iso-Mach surface $(M=1)$, illustrating the supersonic $3 \mathrm{D}$ area along the span (right), $\mathrm{M}=0.73, \mathrm{a}=5^{\circ}, \mathrm{R}=2.63 \times 10^{6}$

Figure 19 shows the wall pressure fluctuations at $75 \%$ of the chord, at mid section of the span in the case of forcing by $100 \mathrm{~Hz}$, (first phase case). It is shown that the selected flap motion leads to an increase of the amplitudes and of the absolute mean values of the pressure fluctuations.

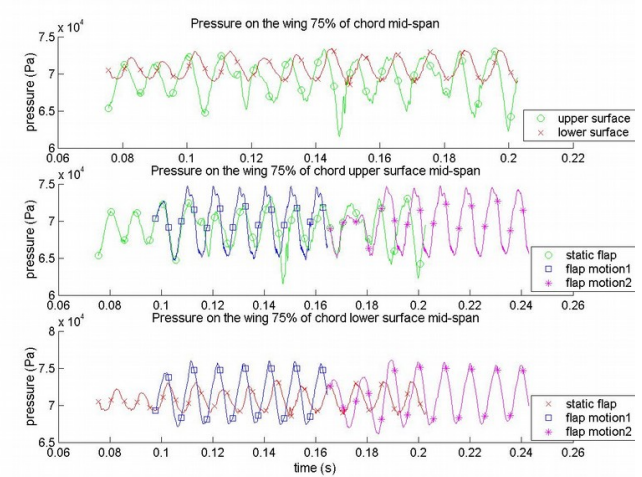

Fig. 19: Wall pressure coefficient oscillations as a function of time at $75 \%$ chord position and at mid-span distance. "Upper surface" means suction side. Motion 1=starting flap motion at minimum of Clift oscillation. Up: Static reference case. Middle: comparison of 
forced case with static case, suction side. Down: comparison of forced case with static case, pressure side, URANS-SA.

Furthermore, this figure shows the influence of the phase concerning the starting of the flap motion, at minimum ("Phase 1") or at maximum ("Phase 2") of lift coefficient respectively. It is found that the buffet mechanism finally follows the phase of the flap motion oscillations.

\section{Conclusions}

A numerical study has been carried out to analyse the transonic interaction around a NACA0012 wing of constant section, including a trailing edge aileron (flap). First, the static case has been analysed and secondly, a forced flap motion case with frequencies equal to the natural buffet and to one third of the buffet frequency. The results are compared with the experimental results of the Institute of Aviation, IoA, provided in the UFAST research program.

A detailed signal processing of the experimental results has been carried out by IMFT by using especially wavelet analysis and Autoregressive (AR) modelling. This treatment was useful to extract the organised frequencies of the buffet phenomenon from the complex chaotic behaviour due to turbulence. A special attention has been attributed to examine the influence of a low amplitude $\left(2^{\circ}\right)$ flap motion.

The numerical simulations have been carried out respecting the exact wind tunnel geometry, by the University of Liverpool (ULIV) and the Institut de Mécanique des Fluides de Toulouse (IMFT). The approaches that have been used in the context of URANS and preliminary DES, show ability of capturing the buffet frequency, as well as the wall pressure coefficient, in a quite good agreement with the experiment. Comparisons were first carried out between the two sets of simulations without knowing the experimental values and afterwards, detailed comparisons with the experiment have been performed. The buffet frequency has been found around $95 \mathrm{~Hz}$.

Concerning the flap motion, both simulations have shown an increase of the amplitudes at forcing equal to the natural buffet frequency. A slight attenuation of the aerodynamic coefficients has been achieved in the case of forcing by one third. This study has shown that the phase response of the system is independent on the initial starting phase of flap motion.

The present study opens a promising way towards a further significant control of the buffet, by using this kind of simulation approaches within an optimisation kernel. In this way, it may be possible to attenuate rather than amplify the oscillations arising from transonic buffet. 


\section{Acknowledgement}

This work was sponsored by the UFAST research program, "Unsteady Effects in Shock Wave Induced separation”, coordinated by IMP-PAN (P. Doerffer). The authors are grateful for the use of the French supercomputing facilities of CINES, IDRIS, GRID-5000.

\section{References}

Barakos, G. UFAST Report, “48-month IoA Case Contribution”, 12 April 2009.

Grossmann, A., Morlet, J., Decomposition of Hardy functions into square integrable wavelets of constant shape, SIAM J., 1984.

Marple S. L., Digital Spectral Analysis Prentice-Hall, Englewood Cliffs, 1987.

Miller M. and Kania V., Parameters for aerofoil and aileron oscillations, UFAST Deliverable D3.1.3, March 2009.

Seegmiller, H. L., Marvin, J. G., And Levy, L L., JR., "Steady and unsteady transonic flow", AIAA J., VOL. 16, N0. 12, Dec.1978, pp. 1260-1270.

Spalart, P. R. and Allmaras, S. R., 1992, "A One-Equation Turbulence Model for Aerodynamic Flows" AIAA Paper 92-0439.

Spalart, P. R., Jou, W.-H., Strelets, M., and Allmaras, S. R. (1997), "Comments on the Feasibility of LES for Wings and on the Hybrid RANS/LES Approach", Advances in DNS/LES, Proceedings of the First AFOSR International Conference on DNS/LES.

Spalart, PR, Deck, S., Shur, M.L., Squires, K.D., Strelets M., Travin, A. 2006. A new version of detached eddy simulation, resistant to ambiguous grid densities. Theor. Comp. Fluid Dyn. 20:181-95

Vos J., Chaput E., Arlinger B., Rizzi A., and Corjon A., 1998 Recent advances in aerodynamics inside the NSMB (Navier-Stokes Multi-Block) consortium. In /36th Aerospace Sciences Meeting and Exhibit/, AIAA Paper 1998-0802, Reno, USA,.

Wilcox, D.C. Turbulence Modelling for CFD, DCW Industries, California, USA, 1994 